\title{
Die Ausscheidung des Salvarsans nach intravenöser Injektion konzentrierter Lösungen.
}

\author{
Von Dr. M. Lewitt in Berlin.
}

In Nr. 14 dieser Wochenschrift berichtet Prof, Dr. Carl Stern (Düsseldorf) über die Dauer der Ausscheidung des Neosalvarsans bei der von ihm zuerst beschriebenen Methode der intravenösen Injektion konzentrierter Lösungen. Das Verfahren selbst ist in der Tat so einfach, daß es ohne geschulte Hilfskräfte leicht auszuführen ist, und ich haba seit 20 Monaten auf der von mir geleiteten Hautstation des Garnison. lazaretts I ausschließlich diese Art der Anwendung bevorzugt.

In der Kriegssitzung der Berliner Dermatologischen Gesellschaft ${ }^{1}$ ) habe ich bereits das Resultat meiner ohemischen Untersuchungen über die Ausscheidung des Neosalvarsans bekanntgegeben. Angewandt wurde gleichfalls die von Abelin beschriebene Methode, wie sie Stern (S. 417) wiedergibt. Nach 48 Stunden war niemals mehr ein Salvarsannachweis im Urin zu erbringen (bei Stern $10 \%$ positiv), auch nach 24 Stứnden nicht in allen Fällen, einige Stunden nach der Injektion dazegen fast immer Rotfärbung der Resorzinprobe.

Daß man bei der Sternschen Methode wohl auf eine gute Wirkung rechnen darf, kann man daraus schließen, daß wir bei der kombinierten Zg-Salvarsankur wesentlich rascher negative Wa.R. erzielten, als es sonst bei ausschließlicher Quecksilberbehandlung der Fall zu sein pflegt.

Zur Nachprüfung der abweichenden Sternschen Resultate werden zurzeit weitere Untersuchungen angestellt. 